\title{
Synthesis of ABA Type Block Copolymers of Poly(Ethylene Glycol) and Poly(Dodecyl Vinyl Ether) and Its Using as Surfactant in Emulsion Polymerization
}

\author{
Yesim Hepuzer Gursel ${ }^{1}$, Ayfer Sarac ${ }^{2 *}$, Bahire Filiz Senkal ${ }^{1}$ \\ ${ }^{1}$ Department of Chemistry, Istanbul Technical University, Istanbul, Turkey \\ ${ }^{2}$ Department of Chemistry, Yildız Technical University, Istanbul, Turkey \\ Email: *ayfersarac2002@yahoo.com
}

Received November 23, 2013; revised December 26, 2013; accepted January 5, 2014

Copyright (c) 2014 Yesim Hepuzer Gursel et al. This is an open access article distributed under the Creative Commons Attribution License, which permits unrestricted use, distribution, and reproduction in any medium, provided the original work is properly cited. In accordance of the Creative Commons Attribution License all Copyrights (C) 2014 are reserved for SCIRP and the owner of the intellectual property Yesim Hepuzer Gursel et al. All Copyright (C) 2014 are guarded by law and by SCIRP as a guardian.

\begin{abstract}
A poly(ethylene glycol) (PEG) based macroinitiator (MI) with terminal chloride atom at both ends was prepared by the reaction of PEG-400 with chloroacetyl chloride and used for the cationic polymerization of dodecyl vinyl ether (DVE) yielding ABA type block copolymer. The block copolymer was then used as the surfactant for the emulsion polymerization of vinyl acetate and styrene in the presence of potassium persulfate as an initiator. The effects of new polymeric emulsifier on the physicochemical properties of obtained latexes were investigated depending on surfactant percentage in homopolymerizations.
\end{abstract}

\section{KEYWORDS}

\section{Cationic Polymerization; Emulsion Polymerization; Poly(Ethylene Glycol); Surfactants}

\section{Introduction}

Emulsion polymerization is a unique chemical process widely used to produce waterborne latexes with various colloidal and physicochemical properties. This heterogeneous free radical polymerization process involves emulsification of the relatively hydrophobic monomer in water by an oil-in-water emulsifier, followed by the initiation reaction with either a water insoluble initiator or an oil-soluble initiator. Typical monomers used in emulsion polymerization include vinyl acetate, butadiene, styrene, acrylonitrile, acrylate methacrylate monomers, and vinyl chloride [1-4].

Heterophase polymerizations, especially emulsion polymerizations, are important industrial technologies yielding synthetic elastomers, paints, paper coatings, adhesives, etc. In emulsion polymerizations, the use, or in situ production, of surfactants, is necessary in order to achieve stabilization of the latex particles produced during polymerization and indeed later on in the derived products [5].

\footnotetext{
${ }^{*}$ Corresponding author.
}

Surfactants are among the most versatile products used in the chemical industry, appearing in such diverse products as motor oils, pharmaceuticals and detergents. More recently, applications have been extended to such high-technology areas as electronic printing, magnetic recording, and biotechnology [6]. There are four classes of surfactants: 1) anionic, where the head group of the molecule has a negative charge; 2) cationic, where the head group bears a positive charge; 3) zwitterionic, where both positive and negative charges are present; and 4) non-ionic, where the head group has no ionic character. Cationic surfactants, which are most relevant to the present study, usually fall into one of the following categories: long-chain amines or polyamines and their respective salts, quarternary ammonium salts (e.g. hexadecyltrimethyl ammonium bromide), oligo (ethylene oxide) amines and their quaternized derivatives, and amine oxides.

Recently, there has been increasing interest in the synthesis of tailor-made polymeric surfactants. The surfactants based on oligomeric and/or polymeric molecules have received wide attraction both at academic and in- 
dustrial scale because of their unique and tailor-made properties over the conventional low molecular weight surface active agents [7]. Although necessarily less welldefined than small-molecule surfactants, polymeric surfactants probably offer greater opportunities in terms of flexibility, diversity and functionality [8]. This is especially true in the light of recent advances in controlled/ living radical polymerization chemistry, as exemplified by atom transfer radical polymerization (ATRP) [9-15] and, to a lesser extent, reversible addition fragmentation transfer (RAFT) polymerization [16]. This new polymer chemistry has enabled synthetic polymer chemists to make new, well-defined amphiphilic block copolymers, many of which exhibit interesting surfactant behavior.

The oligomeric/polymeric surfactants that exhibit association and self-assembly behavior in water are basically amphiphilic in nature and consist of hydrophobic and hydrophilic moieties within the same molecule [17]. Hydrophilic-hydrophobic block copolymers directly prepared in aqueous medium without any additional surfactant are, therefore, of special interest as polymeric surfactants in latex technology [18]. The most effective stabilization of emulsions is obtained using ABA block or $\mathrm{AB}_{\mathrm{n}}\left(\right.$ or $\mathrm{BA}_{\mathrm{n}}$ ) graft copolymers [19]. The B-chain(s) (the "anchor"-chain) is chosen to be highly insoluble in the aqueous medium and strongly adsorbed at (or soluble in) the oil-droplets. The A-chain is chosen to be highly soluble in the aqueous medium and strongly solvated by its molecules [17]. ABA type block copolymers are those based on poly(ethylene oxide) (PEO) and poly(propylene oxide) (PPO), which are also known as Pluronic surfactants, associating in aqueous media into micellar aggregates, and the micellization can either be induced at a given temperature by increasing the concentration beyond a critical value or at a given concentration beyond a critical micelle temperature [7,17]. The hydrophobic moieties, for example, have been either PPO, poly(butylene oxide) (PBO), poly(dimethyl siloxane) (PDMS), polystyrene (PS), or poly(isobutylene) (PIB) [7]. ABA type block copolymers could decrease the surface tension of both water as well as organic oils and, hence, they can be used for surfactant action in organic media, in which conventional low molecular weight surfactants as well as PEO-PPO or PEO-PPO-PEO polymeric surfactants are less effective [7]. Because of the hydrophobic structure at both ends, the ABA type block copolymer synthesized is used as surfactant in this study.

A significant difference in the stabilization mechanism of emulsions based on the Pluronic surfactants can be understood from the conformation of the two polymers at the oil-water interface. With the ABA block copolymer, the PPO B-chain produces small loops at the oil-surface leaving two tails of PEO chains dangling in the aqueous solution. In other words, this type of block copolymer would produce a "brush" at the oil-water interface. The steric repulsion in this case is due to "brush-to-brush" interaction. In contrast, the graft copolymer adsorbs with several alkyl chains in or at the oil-phase leaving strongly hydrated polyfructose loops and small tails in the solution. The steric repulsion in this case is mainly due to "loop-to-loop" interaction [17].

As compared with conventional surfactants, block copolymers in the latex technology may serve not only as a stabilizer in the polymerization process, but also as an active component in the finished product formulation, for example, as plasticizer, compatibilizer, antistatic agent, oil and/or water repellent. The major application possibilities of block copolymer-stabilized dispersions, as well for aqueous and nonaqueous systems, are, therefore, in the fields of coatings, inks, toners, adhesives, and functional packing materials for chromatography [18]. Of all the di- and tri-block copolymer surfactants with PEOPPO, PEO-PPO-PEO have been a subject of intensive investigations, especially for their self-assembly properties, phase behavior, gelation, etc. On the other hand, poly(ethylene glycol) (PEG) is one of the other common hydrophilic polymers studied especially as surfactant, which has potential for application in a variety of fields such as biological and biomedical sciences, surface chemistry and electrochemistry [20]. In spite of some limitations of block copolymers in emulsion polymerization, inherent to their higher molecular weight with respect to classical surfactants, such polymeric surfactants lead, under suitable reaction conditions, to "hairy latexes" where the combination of their hydrophilic fringes and hydrophobic blocks on the particle surface are very attractive. For example, hydrophilic fringes of an adjustable thickness enhance colloidal stability under freezethaw, electrolyte, and shear, reduce foaming, and improve the rheological characteristics [18].

In this study, a macroinitiator with PEO like chain was prepared by the reaction of PEG-400 with chloro acetyl chloride. This initiator was used for cationic polymerization of dodecyl vinyl ether to obtain ABA type block copolymer. This block copolymer was used in the emulsion polymerizations of vinyl acetate (VAc) and styrene (St) as a surfactant, and the effects of this polymeric surfactant quantity were investigated on the main characteristics of poly(vinyl acetate) (PVAc) and polystyrene (PSt) latexes.

\section{Experimental}

\subsection{Materials}

Vinyl acetate (VAc) (Fluka), styrene (St) (Fluka), potassium persulfate (PPS) (Fluka), chloroacetyl chloride (Fluka), poly(ethylene glycol) (PEG-400) (Aldrich), dodecyl vinyl ether (DVE) (Aldrich), AgBF4 (Aldrich) and 
all the chemicals used were analytical grade chemical products.

\subsection{Preparation of Macroinitiator (MI)}

Chloroacetyl chloride ( $44 \mathrm{mmol}, 3.5 \mathrm{~mL}$ ) in $10 \mathrm{~mL}$ of dry THF was added to dropwise to a stirring mixture of triethylamine, (43 mmol, $6 \mathrm{~mL}$ ) and PEG-400 (25 mmol, $10 \mathrm{~g}$ ) in $40 \mathrm{~mL}$ of dry $\mathrm{THF}$ at $0^{\circ} \mathrm{C}$. The reaction was continued under stirring for $18 \mathrm{~h}$ at room temperature. The reaction mixture was filtered, solvent was evaporated and the rest remaining in the mixture was poured in cold diethyl ether. The precipitated was filtered and dried under vacuum. Yield is $13 \mathrm{~g}$.

\subsection{Cationic Polymerization of Dodecyl Vinyl Ether}

The polymerization was carried out under dry nitrogen atmosphere. $10 \mathrm{~mL}$ of DVB was introduced to a threeneck flask heated in vacuo and flushed with dry nitrogen. $1 \mathrm{~g}$ of $\mathrm{MI}$ and $1.05 \mathrm{~g}$ of AgBF4 in $10 \mathrm{~mL}$ of dichloromethane were added under efficient stirring at $-7^{\circ} \mathrm{C}$. After 3 $\mathrm{h}$, metanol was added to the reaction mixture to terminate the polymerization. The polymerization mixture was stirred at room temperature for $24 \mathrm{~h}$. After centrifugation, the polymerization solution was added dropwise into cold methanol. The precipitated polymer was separated by filtration and dried under vacuum at room temperature.

\subsection{Emulsion Polymerization Procedure}

PEG-PDVE block copolymer was used in emulsion polymerization of VAc and St as surfactant. A typical emulsion polymerization was carried out in a $500 \mathrm{~mL}$ three neck glass reactor equipped with a condenser, a mechanical stirrer having a constant speed of $400 \mathrm{rpm}$ and a reflux condenser in a total batch period of $1 \mathrm{~h}$. The polymerization was performed at given temperature different time depending of the surfactant value. The conditions of the polymerizations were given Table 1 . Obtained polymers were precipitated by adding salt and the polymer was filtered and washed with excess of hot water to remove salt and surfactant, and all measurements were carried out these purified latexes.

The effects of surfactant quantity on the physicochemical properties of polymers were investigated by measuring viscosity, viscosity average molecular weight, surface tension, and particle size, by using Brookfield viscometer, viscometric method, du Nouy tensiometer, and particle size analysis, respectively.

\subsection{Measurements}

New synthesized ABA type polymeric surfactant was
Table 1. Formulation used in polymerizations.

\begin{tabular}{ccccccc}
\hline \multirow{2}{*}{ Run } & Monomer & $\begin{array}{c}\text { Monomer } \\
\text { weight (g) }\end{array}$ & $\begin{array}{c}\text { Surfactant } \\
\text { quantity (g) }\end{array}$ & PPS (g) & $\begin{array}{c}\text { Water Temperature } \\
(\mathrm{g})\end{array}$ & $\left({ }^{\circ} \mathrm{C}\right)$ \\
\hline 1 & & & 0.103 & & \\
2 & VAc & 4.00 & 0.260 & 0.080 & 290 & 70 \\
3 & & & 0.360 & & & \\
4 & & & 0.103 & & & \\
5 & St & \multirow{2}{*}{6.80} & 0.260 & 0.075 & 100 & 80 \\
6 & & & 0.360 & & & \\
\hline
\end{tabular}

characterized by FT-IR (Nicholet 380), and ${ }^{1} \mathrm{H}-\mathrm{NMR}$ spectrum was measured on a Bruker Am400 instrument using $\mathrm{CDCl}_{3}$ as the solvent and TMS as internal standard. The number of average molecular weight $\left(\mathrm{M}_{\mathrm{n}}\right)$ of the surfactant was determined by gel permeation chromatography (GPC) by using an Agillant 1100 series consisting of a pump, a RI detector, and Waters styrogel (HR 3 and HR 4) columns. Tetrahydrofuran was used as the eluent and butylated hydroxyl toluene as reference. The flow rate was $1 \mathrm{~mL} / \mathrm{min}$.

Latexes were characterized by measuring Brookfield viscosity, viscosity average molecular weight $\left(\mathrm{M}_{\mathrm{v}}\right)$, surface tension of latexes to air, and particle size. Conversion was monitored gravimetrically. According to the calculating results from solid contents of latexes as theoretical and practical, conversion changed between $86 \%$ and $88 \%$. The original viscosities of the homopolymer latexes were determined by Brookfield Programmable DV-II model viscometer with spindle number S 61 at $23^{\circ} \mathrm{C} . \mathrm{M}_{\mathrm{v}}$ of polymers was determined by capillary intrinsic viscometer at $30^{\circ} \mathrm{C}$. For this purpose, Ubbelohde-type viscometer, acetone and toluene used as solvents for PVAc and PSt respectively. Mark-Houwink constants were used as $\mathrm{a}=0.72$ and $\mathrm{k}=1.02 \times 10^{-3}$ $(\mathrm{mL} / \mathrm{g})$ and $\mathrm{a}=0.73$ and $\mathrm{k}=1.55 \times 10^{-4}(\mathrm{dL} / \mathrm{g})$ for VAc and St respectively. The surface tension measurements were done with a Sigma 701 model tensiometer (KSV instruments, Helsinki, Finland) equipped with a Pt du Nouy ring at $24^{\circ} \mathrm{C}$. The particle size of the latexes was determined by Malvern Zetasizer 6.01 model instrument.

\section{Results and Discussion}

In this study, ABA type tree block copolymer of PEG, PDVE and PEG was prepared by cationic polymerization of dodecyl vinyl ether in the presence of PEG-400 having chlorine groups at both ends as a macroinitiator and a solution of $\mathrm{AgBF}_{4}$ in methylene chloride as catalyst at $-7^{\circ} \mathrm{C}$ for $3 \mathrm{~h}$. The reaction path is represented below (Scheme 1).

The polymeric surfactant was characterized by FT-IR and ${ }^{1} \mathrm{H}$-NMR spectroscopy. According to the Figure 1, in the block copolymer, stretching vibrations occurs at $1740 \mathrm{~cm}^{-1}$ and $2922 \mathrm{~cm}^{-1}-2853 \mathrm{~cm}^{-1}$ belongs to car- 


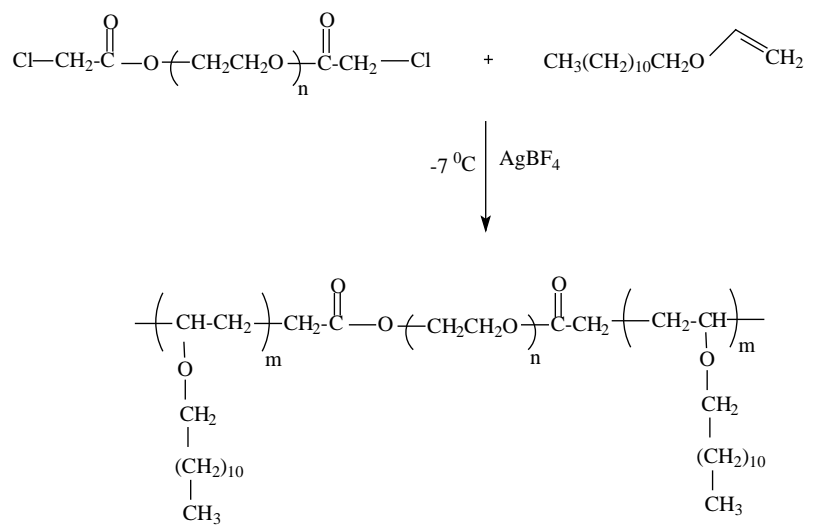

Scheme 1. Synthesis of polymeric surfactant.

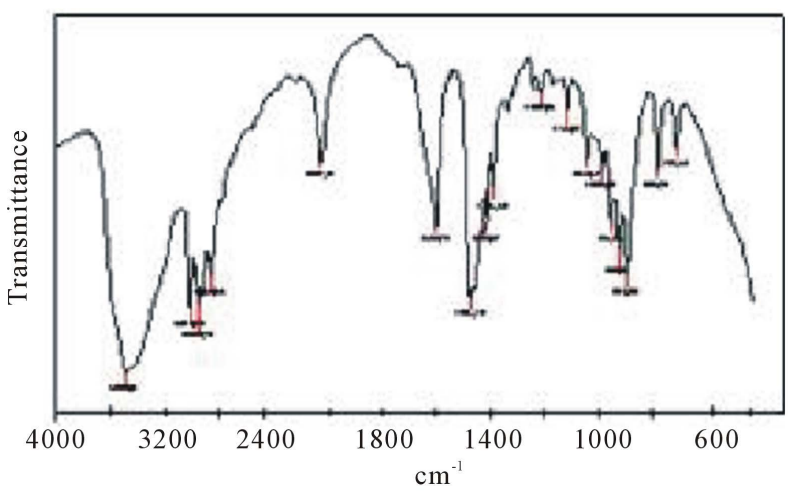

Figure 1. FT-IR spectrum of the polymeric surfactant.

bonyl group and alkyl chains of dodecyl group, respectively.

Also, the C-O stretching vibration occurs at $1054 \mathrm{~cm}^{-1}$ belongs to etheric group. ${ }^{1} \mathrm{H}-\mathrm{NMR}$ spectrum of the polymer was given in Figure 2. Aliphatic protons of alkyl chains of dodecyl groups were observed as a multiplet at 1.20 - $1.80 \mathrm{ppm}$ and etheric protons of PEG were observed at $3.0-4.0 \mathrm{ppm}$.

The number average molecular weight and polydispersity of the polymeric surfactant were detected as 1152 $\mathrm{g} / \mathrm{mol}$ and 1.10 respectively (Figure 3 ). The narrow molecular weight clearly show that the polymerization quantitatively proceeds in a controlled fashion to afford a polymer with well-defined chain structure under the condition employed.

\subsection{Viscosity}

Dispersion with a large number of small particles exhibits a higher viscosity than one with a small number of large particles [21]. Polymeric surfactant accelerated the reaction, because of increasing with the length of the hydrophobic part of the surfactant. Increasing surfactant concentration in the polymer recipe slightly increased the latex viscosities, but it does not affect very seriously on the viscosities of VAc and St latexes

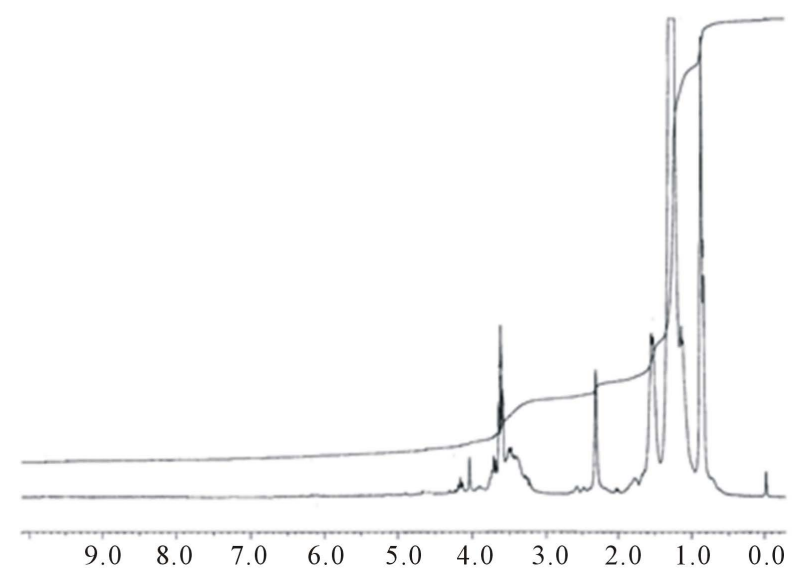

Figure 2. ${ }^{1} \mathbf{H}$-NMR spectrum of the polymeric surfactant.

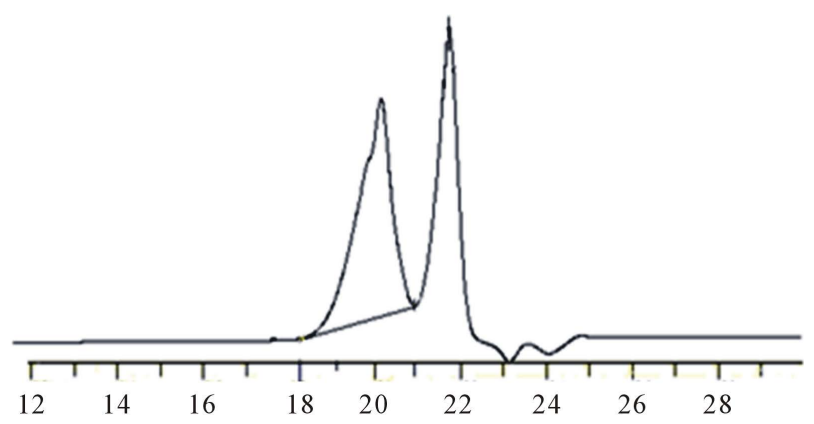

Figure 3. GPC traces of the polymeric surfactant.

\section{(Table 2).}

The main advantages of block copolymer synthesis in emulsion, over conventional bulk or solution polymerizations, are that such polymerizations do not require drastic experimental conditions such as anionic or cationic "living polymerizations". Moreover, free radical emulsion polymerizations are environmentally friendly methods for the production of polymers due to the absence of volatile organic compounds. These polymerizations often reach very high conversions; the heat produced by exothermic reactions is dissipated by the water and high-molecular weight polymers can be obtained in a low viscosity media. The final product can thus be directly used in its latex form, in coating applications for instance [18].

\subsection{Surface Tension}

The surface tension measurements were performed on a Sigma 701 model tensiometer (KSV instruments, Helsinki, Finland) equipped with a $\mathrm{Pt}$ du Nouy ring at $24^{\circ} \mathrm{C}$, and results were given in Table 2 . Surface tension of homopolymer latexes increased with surfactant concentration. This can be explained by taking into account that the surface area stabilized by the surfactant increases with an increase of the concentration of the surfactant 
Table 2. Colloidal characteristics of latexes.

\begin{tabular}{ccccccc}
\hline Run Monomer & $\begin{array}{c}\text { Surfactant } \\
\text { quantity } \\
(\mathrm{g})\end{array}$ & $\begin{array}{c}\text { Brookfield } \\
\text { viscosity } \\
(\mathrm{cP})\end{array}$ & $\begin{array}{c}\text { Surface } \\
\text { tension } \\
(\mathrm{mN} / \mathrm{m})\end{array}$ & $\begin{array}{c}\mathrm{M}_{\mathrm{v}} \\
(\mathrm{g} / \mathrm{mol})\end{array}$ & $\begin{array}{c}\text { Particle } \\
\text { size } \\
(\mathrm{nm})\end{array}$ \\
\hline 1 & & 0.103 & 2.04 & 27.72 & 88.274 & 191.7 \\
2 & VAc & 0.260 & 2.34 & 30.53 & 104.712 & 187.3 \\
3 & & 0.360 & 2.76 & 38.88 & 117.177 & 182.8 \\
4 & & 0.103 & $<1.5$ & 30.67 & 45.000 & 428.6 \\
5 & St & 0.260 & $<1.5$ & 36.41 & 65.956 & 379.5 \\
6 & & 0.360 & $<1.5$ & 40.67 & 89.334 & 363.2 \\
\hline
\end{tabular}

resulting in smaller particles due to mass balance considerations. The increasing hydrophobic character into polymer causes to increasing emulsifier adsorption onto polymer particles. Thus the increasing of free emulsifier concentration in latex by blocking of emulsifier adsorption and the decreasing of polarity differences between interfaces cause to increase the surface tension, especially for VAc latexes.

Surface tension can be used as a measure for the coverage of particles. As can be derived from the surface tension values, the particle surfaces of the latexes are incompletely covered with surfactant molecules because the surface tensions of the latexes lie well above the values of the saturated surfactant solution. The smaller the particles are, the higher the surface tension is and, therefore, the coverage of the particles with surfactant increases with decreasing particle size [22].

\subsection{Particle Size}

The particle size of the final latexes was determined by Zetasizer, and can be seen in Table 2. It was found that the particle size mainly depends on the amount of surfactant in both latex series. The presence of the long hydrophobic chain of the Pluronic surfactants had a screening effect on the Coulombic forces which prevents the surfactant adsorption. Consequently, the higher the chain lengths of the hydrophobic part of the surfactant, the higher the surfactant adsorption on the polymer [23]. The polymeric surfactant causes to decrease the number of particles in the volume unit, thus viscosity of latexes increased, and particle size decreased.

An increase of the surfactant quantity from 0.103 to 0.360 (wt) results in a decrease of the particle size and an increase viscosity. The viscosity of the dispersions measured increases drastically (Table 2). This can be explained by taking into account that the surface area stabilized by the surfactant increases with an increase of the concentration of the surfactant resulting in smaller particles due to mass balance considerations. A dispersion with a large number of small particles exhibits a higher viscosity than one with a small number of large particles [21].

\section{Conclusion}

In this study, commercially available PEG-400 was modified to prepare macroinitiator which was then used in the cationic polymerization of dodecyl vinyl ether. The new surfactant was used in emulsion polymerization of vinyl acetate and styrene. It was determined that when the concentration of the polymeric surfactant was increased, the resulting latex viscosity slightly increases, particle size decreases regularly, and the viscosity molecular weight of final latex increases. The surface tension and particle size of polymer latex follow different trends with the increasing of the surfactant concentration. Meanwhile, they were changed very seriously. It was found that the surfactant concentration was sensitive to such parameters as viscosity, molecular weight, surface tension, and particle size.

\section{Acknowledgements}

This work were supported by the Turkish Scientific and Technological Research Council (TUBITAK) (Project Number: 108T722) and Scientific Research Projects Coordination Center of Yildiz Technical University (Project Number: 2012-01-02-KAP04).

\section{REFERENCES}

[1] F. A. Bovey, I. M. Kolthoff, A. I. Medalia and E. J. Meehan, "Emulsion Polymerization," Interscience Publishers, New York, 1965.

[2] D. C. Blackley, "Emulsion Polymerization. Theory and Practice,” Applied Science, London, 1975.

[3] R. M. Fitch, "Polymer Colloids: A Comprehensive Introduction,” Academic Press, London, 1997.

[4] C. S. Chern, "Emulsion Polymerization Mechanisms and Kinetics,” Progress in Polymer Science, Vol. 31, No. 5, 2006, pp. 443-486.

http://dx.doi.org/10.1016/j.progpolymsci.2006.02.001

[5] A. Montoya-Goni, D. C. Sherrington, H. A. S. Schoonbrood and J. M. Asua, "Reactive Surfactants in Heterophase Polymerization. XXIV. Emulsion Polymerization of Styrene with Maleate- and Succinate-Containing Cationic Surfactants,” Polymer, Vol. 40, No. 6, 1999, pp. 13591366.

[6] M. J. Rosen, "Surfactants and Interfacial Phenomena," 2nd Edition, Wiley Interscience, New York, 1989.

[7] S. S. Soni, N. V. Sastry, V. K. Aswal and P. S. Goyal, "Micellar Structure of Silicone Surfactants in Water from Surface Activity, SANS and Viscosity Studies," The Journal of Physical Chemistry B, Vol. 106, No. 10, 2002, pp. 2606-2617. http://dx.doi.org/10.1021/jp0129434

[8] S. Liu and S. P. Armes, "Recent Advances in the Synthesis of Polymeric Surfactants," Current Opinion in Colloid \& Interface Science, Vol. 6, No. 3, 2001, pp. 249-256. http://dx.doi.org/10.1016/S1359-0294(01)00090-5

[9] J. S. Wang and K. Matyjaszewski, “Controlled 'Living' 
Radical Polymerization. Atom Transfer Radical Polymerization in the Presence of Transition-Metal Complexes,” Journal of the American Chemical Society, Vol. 117, No. 20, 1995, pp. 5614-5615.

http://dx.doi.org/10.1021/ja00125a035

[10] M. Kato, M. Kamagaito, M. Sawamoto and T. Higashimura, "Polymerization of Methyl Methacrylate with the Carbon Tetrachloride/Dichlorotris-(triphenylphosphine) ruthenium(II)/Methylaluminum Bis(2,6-di-tert-butyl phenoxide) Initiating System: Possibility of Living Radical Polymerization,” Macromolecules, Vol. 28, No. 5, 1995, pp. 1721-1723. http://dx.doi.org/10.1021/ma00109a056

[11] T. E. Patten and K. Matyjaszewski, "Atom Transfer Radical Polymerization and the Synthesis of Polymeric Materials,” Advanced Materials, Vol. 10, No. 12, 1998, pp. 901-915.

http://dx.doi.org/10.1002/(SICI)1521-4095(199808)10:12 $<901:$ AID-ADMA901>3.0.CO;2-B

[12] K. Matyjaszewski, J. Qiu, N. V. Tsarevsky and B. Charleux, "Atom Transfer Radical Polymerization of n-Butyl Methacrylate in an Aqueous Dispersed System: A Miniemulsion Approach," Journal of Polymer Science Part A: Polymer Chemistry, Vol. 38, No. S1, 2000, pp. 47244734.

http://dx.doi.org/10.1002/1099-0518(200012)38:1+<4724 :AID-POLA120>3.0.CO;2-Q

[13] D. H. Haddleton, S. Perrier and S. A. F. Bon, "Copper (I)-Mediated Living Radical Polymerization in the Presence of Oxyethylene Groups: Online H-1 NMR Spectroscopy to Investigate Solvent Effects," Macromolecules, Vol. 33, No. 22, 2000, pp. 8246-8251. http://dx.doi.org/10.1021/ma001097c

[14] X. S. Wang and S. P. Armes, "Facile Atom Transfer Radical Polymerization of Methoxy-Capped Oligo(Ethylene Glycol) Methacrylate in Aqueous Media at Ambient Temperature," Macromolecules, Vol. 33, No. 18, 2000, pp. 6640-6647. http://dx.doi.org/10.1021/ma000671h

[15] F. Zeng, Y. Shen, S. Zhu and R. Pelton, “Atom Transfer Radical Polymerization of 2-(Dimethylamino)Ethyl Methacrylate in Aqueous Media," Journal of Polymer Science Part A: Polymer Chemistry, Vol. 38, No. 20, 2000, pp. 3821-3827. http://dx.doi.org/10.1002/1099-0518(20001015)38:20<38 21::AID-POLA130>3.0.CO;2-G

[16] G. Moad, J. Chiefari, Y. K. Chong, J. Krstina, R. T. A. Mayadunne, A. Postma, E. Rizzardo and S. H. Thang,
"Living Free Radical Polymerization with Reversible Addition-Fragmentation Chain Transfer (the Life of RAFT),” Polymer International, Vol. 49, No. 9, 2000, pp. 993-1001.

http://dx.doi.org/10.1002/1097-0126(200009)49:9<993:: AID-PI506>3.0.CO;2-6

[17] D. Exerowaa, G. Gotcheva, T. Kolarova, K. Kristova, B. Leveckeb and T. Tadros, "Comparison of Oil-in-Water Emulsion Films Produced Using $\mathrm{ABA}$ or $\mathrm{AB}_{\mathrm{n}}$ Copolymers," Colloids and Surfaces A: Physicochem Eng Aspects, Vol. 335, No. 1, 2009, pp. 50-54. http://dx.doi.org/10.1016/j.colsurfa.2008.10.025

[18] G. Riess and C. Labbe, "Block Copolymers in Emulsion and Dispersion Polymerization," Macromol Rapid Commun, Vol. 25, No. 2, 2004, pp. 401-435. http://dx.doi.org/10.1002/marc.200300048

[19] J. Zhang, M. R. Dubay, C. J. Houtman and S. J. Severtson, "Sulfonated Amphiphilic Block Copolymers: Synthesis, Self-Assembly in Water, and Application as Stabilizer in Emulsion Polymerization,” Macromolecules, Vol. 42, No. 14, 2009, pp. 5080-5090. http://dx.doi.org/10.1021/ma900795f

[20] Y. J. Kim, Y. Nagasaki, K. Kataoka, M. Kato, M. Yokoyama, T. Okano and Y. Sakurai, "Heterobifunctional Poly(Ethylene Oxide)-One-Pot Synthesis of Poly(Ethylene Oxide) with a Primary Amino Group at One End and a Hydroxyl Group at the Other End,” Vol. 33, No. 10, 1994, pp. 1-6.

[21] C. Heldmann, R. I. Cabrera, B. Momper, R. Kuropka and K. Zimmerschied, "Influence of Nonionic Emulsifiers on the Properties of Vinyl Acetate/VeoVa10 and Vinyl Acetate/Ethylene Emulsions and Paints," Progress in Organic Coatings, Vol. 35, No. 1, 1999, pp. 69-77. http://dx.doi.org/10.1016/S0300-9440(99)00025-9

[22] K. Landfester, "Polyreactions in Miniemulsions," Macromolecular Rapid Communications, Vol. 22, No. 12, 2001, pp. 896-936.

http://dx.doi.org/10.1002/1521-3927(20010801)22:12<89 6::AID-MARC896>3.0.CO;2-R

[23] D. H. Napper and A. E. Alexander, "Polymerization of Vinyl Acetate in Aqueous Media. Part II. The Kinetic Behavior in the Presence of Low Concentrations of Added Soaps,” Journal of Polymer Science, Vol. 61, No. 171, 1962, pp. 127-133.

http://dx.doi.org/10.1002/pol.1962.1206117116 